\title{
Epidemiological Study on Cerebrovascular Disease in a Japanese Community, Hisayama
}

\author{
Kazuo Ueda \\ School of Health Science and Second Department of Internal Medicine, \\ Faculty of Medicine, Kyushu University, Fukuoka
}

\begin{abstract}
Incidence and risk factors for cerebral stroke and its changing pattern with time were studied in a prospective population survey which has been conducted in a Japanese rural community, Hisayama. Cerebral infarction was more frequent than intracerebral hemorrhage among Hisayama residents, however, the incidence of intracerebral hemorrhage would actually have been higher in Hisayama residents than in western populations especially in 1960 's or 1970's. Incidence of both intracerebral hemorrhage and cerebral infarction decreased in the recent Hisayama population, and among the types of cerebral infarction perforating infarcts most prominently decreased. The reduced prevalence of hypertension and successful management of hypertension were considered to contribute to the reduction in cerebral stroke. It is expected, however, that characteristics or patterns of cerebral stroke would change in the future with increasing prevalence of atherogenic factors among the Japanese population.

Cerebral stroke, Risk factors, Lacunar infarcts, Hypertension, Hisayama study
\end{abstract}

\section{INTRODUCTION}

In this paper I describe characteristics of cerebrovascular disease (cerebral stroke) for Japanese in the following aspects based on the results obtained from the long-term prospective study, which has been carried out since 1961 in the Japanese rural area, Hisayama town, Fukuoka province: 1) type-or subtype-specific incidence of cerebral stroke and risk factors observed during 22-year follow-up period; 2) change in the incidence of cerebral stroke and its related factors, which has been obtained from comparison of two Hisayama populations over a 13-year period. 


\section{OUTLINE OF THE HISAYAMA STUDY}

The Hisayama study is fundamentally a prospective cohort study. One thousand and six hundred twenty-one subjects of both sexes aged 40 or over who had never suffered cerebral stroke were recruited from the residents (about $90 \%$ of the total population of the same agerange) in 1961 as a cohort, and they have prospectively been followed-up since then with only $0.5 \%$ lost (first cohort). Newly occurred cerebral stroke was detected by clinical examination of study physicians including ancillary diagnostic procedures such as lumbar puncture, cerebral angiography or recently computed tomography. This could be achieved through a tight daily monitoring between the study center and local clinics or the central town office. The most striking feature of the study is that causes of death were examined by autopsy in about $80 \%$ of the deceased. The autopsy examination was also usable to revise ante-mortem diagnosis and to obtain more detailed information on underlying diseases. ${ }^{(1)}$ After 13-year follow-up, we selected 2,053 subjects of both sexes aged 40 or over in 1974 as the new cohort aimed at knowing changing pattern of the disease and related factors (second cohort). They have also been followed-up since then in the same manner as that for the first cohort without loss. ${ }^{(2)}$ The sex \& age composition of two cohorts was almost identical.

\section{RESULTS AND COMMENTS}

Table 1 indicates the type-specific frequency of cerebral stroke for the first cohort during 22 years of follow-up. Of 292 newly occurred cerebral strokes cerebral infarction (CI) was the most frequent (71.6\%). Intracerebral hemorrhage (ICH) accounted for $16.8 \%$, subarachnoid hemorrhage (SAH) for $8.9 \%$, and poorly defined types for $2.7 \%$. Table 2 reveals average annual incidence of ICH and CI for the first cohort by sex and age-decades during 22-year period between 1961 and 1983. CI was more frequent than ICH in each decade of age for both sexes, and the rate for $\mathrm{CI}$ increased with advancing age-decade. Totally ICH was $2.2 / 1,000 / \mathrm{yr}$ for men and 0.7 for women, $\mathrm{CI} 7.0$ and 5.6, respectively. Thus ratio of $\mathrm{CI}$ to $\mathrm{ICH}$ was 3.2 for men and 6.7 for women. ${ }^{(3)}$ Two points concerned can be discussed from this evidence. Firstly, whether or not $\mathrm{ICH}$ is more frequent in Japanese than in western population as previously expected. Data on proportional frequency of ICH among all types of cerebral stroke can be obtained from surveys of stroke registries, which have been recently conducted in the United States and Europe. ICH caused 4-11\% of all strokes in the Harvard Cooperative Stroke Registry, National Stroke Survey, Stroke Data Bank, and community hospital-based stroke programs. Frequency of ICH in the community-based stroke registries in Sweden, the Netherlands, and Finland was approximately $10 \%$. Some of those studies are based on hospital records as the source of data and may be selectively biased by emphasizing strokes that are severe and required hospitalization; general population survey data can be more representative. According to the results from Framingham Study for a 26-year follow-up, the frequency of ICH among total stroke was 5\% for men and 3\% for women. The Framingham data also show that nine ICHs developed over 24 years in an original population of 2,282 men and seven in a group of 2,845 women, thus permitting an estimated crude incidence of $\mathrm{ICH}$ of $0.16 / 1,000 / \mathrm{yr}$ for men and $0.10 / 1,000 / y r$ for women. Comparing with these figures, $\mathbf{I C H}$ was observed more frequently in our study with the crude incidence $2.2 / 1,000 / \mathrm{yr}$ and $0.7 / 1,000 / \mathrm{yr}$ respectively. This evidence suggests that the notably higher mortality from $\mathrm{ICH}$ indicated by the vital statistics of Japan in 1950 reflects both bias originating from the death-certificate practice of Japanese physicians and from the higher incidence of ICH. Another point to be discussed is the 
Table 1 Type-Specific Frequency of Cerebral Stroke, First Cohort, Hisayama, 1961-1983

\begin{tabular}{|c|c|c|c|c|c|c|}
\hline & \multicolumn{2}{|c|}{ Men } & \multicolumn{2}{|c|}{ Women } & \multicolumn{2}{|c|}{ Total } \\
\hline & No. & $\%$ & No. & $\%$ & No. & $\%$ \\
\hline Cerebral infarction* & 109 & 71.7 & 100 & 71.4 & 209 & 71.6 \\
\hline Intracerebral hemorrhage & 34 & 22.4 & 15 & 10.7 & 49 & 16.8 \\
\hline Subarachnoid hemorrhage & 6 & 3.9 & 20 & 14.3 & 26 & 8.9 \\
\hline Ill-defined & 3 & 2.0 & 5 & 3.6 & 8 & 2.7 \\
\hline Total & 152 & 100.0 & 140 & 100.0 & 292 & 100.0 \\
\hline
\end{tabular}

*Embolic and nonembolic stroke.

Table 2 Average Annual Incidence Rates of Cerebral Infarction and Intracerebral Hemorrhage by Sex and Age at Entry, First Cohort, Hisayama, 1961-1983

\begin{tabular}{|c|c|c|c|c|c|c|c|c|c|c|}
\hline \multirow{3}{*}{$\begin{array}{l}\text { Age at } \\
\text { entry }\end{array}$} & \multicolumn{5}{|c|}{ Men } & \multicolumn{5}{|c|}{ Women } \\
\hline & \multirow{2}{*}{$\begin{array}{l}\text { No. of } \\
\text { subjects }\end{array}$} & \multicolumn{2}{|c|}{$\mathrm{CI}$} & \multicolumn{2}{|c|}{$\mathrm{ICH}$} & \multirow{2}{*}{$\begin{array}{c}\text { No. of } \\
\text { subjects }\end{array}$} & \multicolumn{2}{|c|}{$\mathrm{Cl}$} & \multicolumn{2}{|c|}{$\mathrm{ICH}$} \\
\hline & & Rate & Cases & Rate & Cases & & Rate & Cases & Rate & Cases \\
\hline $40-49$ & 246 & 2.8 & 15 & 1.1 & 6 & 305 & 1.0 & 7 & 0.9 & 6 \\
\hline $50-59$ & 216 & 6.3 & 30 & 3.2 & 15 & 263 & 4.1 & 24 & 0.9 & 5 \\
\hline $60-69$ & 168 & 10.8 & 40 & 2.4 & 9 & 194 & 8.0 & 34 & 0.2 & 1 \\
\hline $70+$ & 77 & 14.2 & 24 & 2.4 & 4 & 152 & 10.5 & 35 & 0.9 & 3 \\
\hline Total & 707 & 7.0 & 109 & 2.2 & 34 & 914 & 5.6 & 100 & 0.7 & 15 \\
\hline
\end{tabular}

Cl. cerebral infarction; ICH, intracerebral hemorthage; rate, cases $1.000 / y r$. $\mathrm{CI}$ : ICH for men 3.2. for women 6.7 .

low incidence of ICH for women in our population. This is much different from ICH mortality figures in the nation-wide statistics. Comparing diagnosis of $\mathrm{ICH}$ on death certificates with autopsy diagnosis, about $30 \%$ of SAH in autopsy cases for women were misdiagnosed as $\mathrm{ICH}^{(4)}$ In the Hisayama study, incidence of SAH increased with advancing age-decade and was much higher than that previously reported from western populations. ${ }^{(5)}$ Therefore, it is suggested that incidence of SAH for women might be higher than expected and SAH might likely be misdiagnosed as ICH, and this probably increases mortality figures from ICH in the vital statistics.

Hypertension was the most important predisposing factor for ICH. In addition, ECG evidence suggesting left ventricular hypertrophy was also considered as a risk factor for $\mathrm{ICH}^{(3)}$, and it appears that heart weight is more closely related to the duration of hypertension. Ueshima et al. reported that low serum total cholesterol was strongly associated with ICH incidence in a community of northern Japan. In our study ${ }^{(3)}$, both high and low cholesterol levels seemed to be risk factors. Several possible explanations can be drawn from our study. Serum total cholesterol has no effect on the development of $\mathrm{ICH}$, and an association of serum cholesterol with ICH mortality may be temporal and not causative. It is quite conceivable that some additional factor(s) may account for the observed association of serum cholesterol with ICH mortality.

Aging and hypertension were important as a risk factor for CI. Abnormal findings in the ocular fundi, high R or ST-T changes on ECG also contributed to the development of CI in our study. ${ }^{(6)} \mathrm{CI}$ can be classified into three types according to the location or cause of the lesions: 1) infarcts on perforating arteries; 2) cortical infarcts (atherothrombotic brain infarcts); 3) cerebral embolism. Table 3 shows the frequency of $\mathrm{ICH}$ and the three types of $\mathrm{CI}$ reported from community studies or stroke registration studies. ${ }^{(7)}$ Subarachnoid hemorrhage or unspecified origin of infarction is not included in the table. The frequency of perforating infarcts 
(lacunar infarcts) was much higher in the Hisayama study than in studies from western countries. ICH was also more frequent in the Hisayama study. The risk factors among three types of CI in the Hisayama study are studied. Lacunar infarcts were more frequently associated with hypertension, glucose intolerance and atrial fibrillation. Cortical infarcts were independently associated with hypertension and cerebral embolism with atrial fibrillation. It is yet uncertain why lacunar infarcts were strongly related to atrial fibrillation, but this observation is noteworthy because it suggests that hypertension exerts an important effect, i.e. the production of lesions on intracerebral small arteries as well as aggravating atherosclerosis. By comparing the incidence of cerebral stroke in the first and the second cohort populations, the change with time in stroke incidence among the Hisayama residents can be assessed. The incidence of ICH decreased in the second cohort in men less than 70 years old, but tended to increase in those aged 70 or more. The age-adjusted incidence for all age groups decreased significantly in the second cohort for men, but not for women because of the small number of cases of ICH. ${ }^{(3)} \mathrm{CI}$ tended to decrease in the second cohort in all age groups and both sexes, and the difference in incidence between the two periods was most significant in the 60-to-70-yearold men. ${ }^{(8)}$ The prevalence of relevant risk factors was compared between two cohorts at the start of follow-up. The frequency of hypertension as well as a higher R-waves on ECG (Minnesota code $\mathrm{III}_{1}$ ) was lower in men in the second cohort; the frequencies of hypercholesterolemia (total cholesterol $>200 \mathrm{mg} / \mathrm{dl}$ ) and obesity significantly increased for both sexes in the second cohort. ${ }^{(2)}$ The reduction in incidence of ICH might be related in particular to the decreased prevalence of hypertension. An action program for the management of hypertension has been in existence since 1975 in Hisayama. A comparison of the average values of blood pressure during follow-up of the first and the second cohort showed that the reduction in

Table 3 Frequency of selected cerebral infarstion by types and intracerebral hemorrhage reported in the present and previous studies

\begin{tabular}{|c|c|c|c|c|c|c|}
\hline \multirow{2}{*}{ Studies } & \multirow{2}{*}{ Period } & \multirow{2}{*}{$N$} & \multicolumn{3}{|c|}{ Infarction (\%) } & \multirow{2}{*}{$\begin{array}{c}\text { Intracerebral } \\
\text { hemorrhage } \\
\left(\begin{array}{c}0 \\
0\end{array}\right)\end{array}$} \\
\hline & & & Perforating & Cortical & Embolic & \\
\hline \multicolumn{7}{|l|}{ Present study } \\
\hline First cohort & $1961-74$ & 174 & 49 & 13 & 12 & 21 \\
\hline Second cohort & $1974-87$ & 163 & 46 & 14 & 20 & 17 \\
\hline \multicolumn{7}{|l|}{ Harvard Cooperative } \\
\hline Stroke Registry, USA') & 1972-? & 649 & 20 & 36 & 33 & 11 \\
\hline Pilot Stroke Bank, USA ${ }^{\text {t1) }}$ & $1980-81$ & 809 & 12 & 21 & 25 & 12 \\
\hline South Alabama, USA") & 1980 & 151 & 13 & 48 & 28 & 9 \\
\hline \multicolumn{7}{|l|}{ Lausanne Stroke } \\
\hline Registry, Switzerland" & 1982-? & 1000 & $13(15)$ & $39(43)$ & $16(20)$ & 11 \\
\hline Austin, Australia") & $1977-80$ & 616 & 21 & 32 & 8 & 6 \\
\hline
\end{tabular}

Cases with mixed etiology included in parentheses.

") Mohr, J. P., et al., Neurology, 1978; b) Kunitz, S. C., et al., Stroke, 1984: ') Gross. C. R., et al.. Stroke. 1984:

() Bogousslavsky, J., et al.. Stroke. 1988: ") Chambers, B. R., et al., Aust. N.Z. J. Med., 1983.

systolic and diastolic pressure was greater in both mild hypertensive and hypertensive people in the second cohort. During the study period, the incidence of $\mathrm{ICH}$ and $\mathrm{CI}$ also markedly decreased in mild hypertensive and hypertensive people from the second cohort. ${ }^{(9)}$ It is difficult to define a clear benefit from controlling hypertension in this community-based programme, because the changes in stroke incidence may have resulted from social, economic, environmental, or nutritional factors that are usually not adequately measured in longitudinal epidemiological studies. Figure 1 shows the incidence of the three types of $\mathrm{CI}$ in the two study 
populations from the Hisayama study. ${ }^{(10)}$ It can be seen that only perforating infarcts decreased significantly in the second cohort. Perforating infarcts are more closely related to hypertensive vascular lesions than to atherosclerosis. This observation is also noteworthy, therefore, because it suggests that improvement in blood pressure control in the second period of the Hisayama study prevented the progression of small arterial lesions in the brain, resulting in a reduction in ICH and CI. A pathological study also found less frequent fibrinoid necrosis in small intracerebral arteries in the more recent autopsy cases. ${ }^{(1)}$ Angiographic studies of native Japanese populations have reported fewer extracranial than intracranial lesions, a reversal of the pattern found in Caucasians. It might be assumed from this finding that transient ischemic attacks (TIAs) would be less frequent in Japanese than in Caucasian people. The incidence and prevalence of TIAs in the Hisayama study were in fact lower than in the western populations. It should be noted that a percentage of TIAs in Japanese may be caused by small infarctions in the basal ganglia. ${ }^{(12)}$
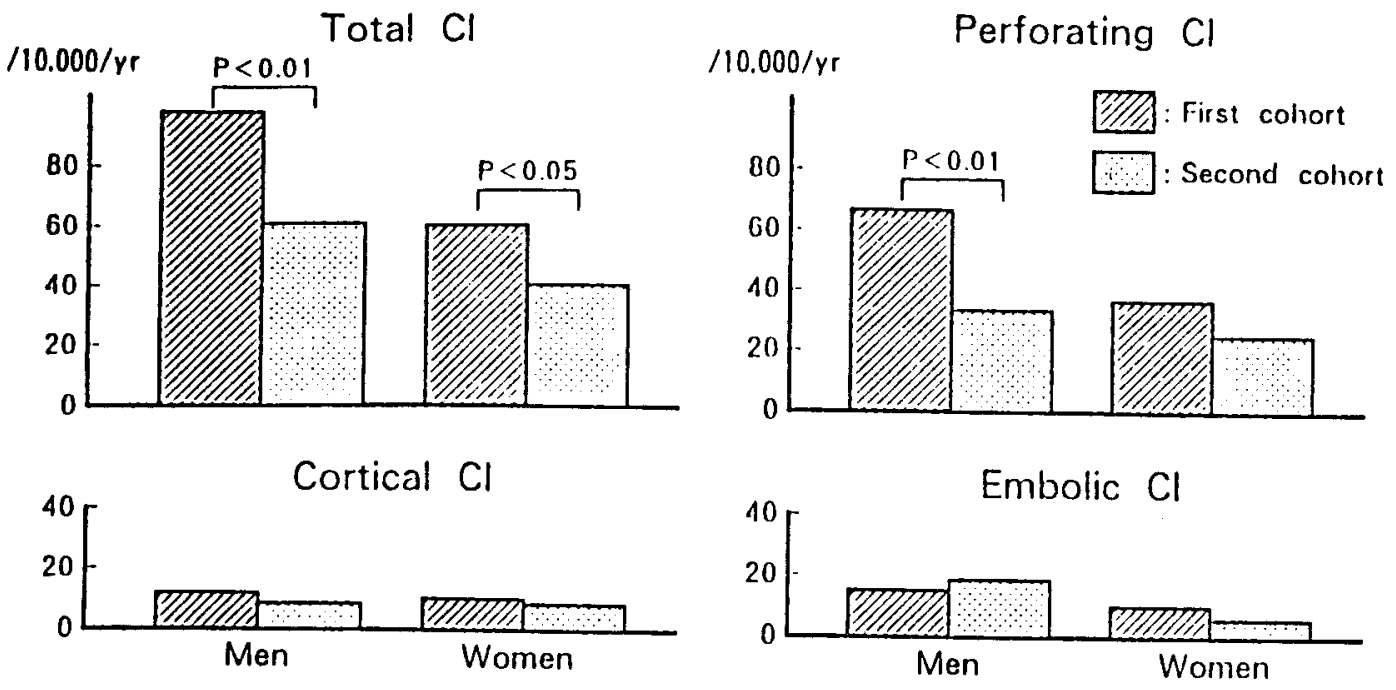

Figure 1 Comparison of age-adjusted average annual incidence rates of cerebral infarction by types between two Hisayama cohorts.

\section{REFERENCES}

1. Ueda, K., Omae, T., Hirota, Y., et al: Decreasing trend in incidence and mortality from stroke in Hisayama residents, Japan. Stroke, 12: 154, 1981.

2. Ueda, K., Omae, T.: Control of hypertension in a Japanese community, In Handbook of Hypertension, ed., Bulpitt, C.J., vol 6. Epidemiology of Hypertension. Amsterdam, Elsever Science Publishers, p. 412, 1985.

3. Ueda, K., Hasuo, Y., Kiyohara, Y., et al.: Intracerebral hemorrhage in a Japanese community, Hisayama: Incidence, changing pattern during long-term follow-up, and related factors. Stroke, 19: 48, 1988.

4. Hasuo, Y., Ueda, K., Kiyohara, Y., et al: Accuracy of diagnosis on death certificates for underlying causes of death in a long-term autopsy-based population study in Hisayama, Japan: With special reference to cardiovascular disease. J. Clin. Epidemiol., 45: 577, 1989.

5. Kiyohara, Y., Ueda, K., Hasuo, Y., et al: Incidence and prognosis of subarachnoid hemorrhage in a Japanese rural community. Stroke 20: 1150, 1989. 
6. Omae, T., Ueda, K.: Risk factors of cerebral stroke in Japan: Prospective epidemiological study in Hisayama community, In Proceedings of 12th World Cogress of Neurology, eds., Katsuki,S., Tsubaki,T., Toyokura,Y., Amsterdam, Excerpta Medica, p. 119, 1982.

7. Ueda, K.: Change in the frequency and type of cerebrovascular disease in Japan. ACE REPORT 75: 5, 1991.

8. Omae, T., Ueda, K.: Editorial review. Hypertension and cerebrovascular disease - the Japanese experience. J. Hypertens., 6:343, 1988.

9. Ueda, K., Omae, T., Hasuo, Y., et al: Prevalence and long-term prognosis of mild hypertensives and hypertensives in a Japanese community, Hisayama. J. Hypertens., 6: $981,1988$.

10. Ueda, K.: Special lecture. Atherosclerosis and cardiovascular disease observed in a Japanese rural community, Hisayama (in Japanese with English abstract). J. Jpn. Atheroscler. Soc., 19: 705, 1991.

11. Masuda, J., Tanaka, K., Omae, T., et al: Cerebrovascular disease and their underlying vascular lesions in Hisayama, Japan - A pathological study of autopsy cases. Stroke, 14: 934, 1983.

12. Ueda, K., Kiyohara, Y., Hasuo, Y., et al: Transient cerebral ischer:c attacks in a Japanese community, Hisayama, Japan. Stroke, 18: 844, 1987. 